\title{
Causal Recursive Parameter Estimation for Discrete-Time Hidden Bivariate Markov Chains
}

\author{
Yariv Ephraim, Fellow, IEEE, and Brian L. Mark, Senior Member, IEEE
}

\begin{abstract}
An algorithm for causal recursive parameter estimation of a discrete-time hidden bivariate Markov chain is developed. In this model, a discrete-time bivariate Markov chain is observed through a discrete-time memoryless channel. The algorithm relies on the EM-based recursive approach developed by Stiller and Radons for hidden Markov models. A distinct advantage of the discrete-time hidden bivariate Markov chain model is that the sojourn time distribution of its observable process in each state is phase-type rather than geometric as in the hidden Markov model. Phase-type distributions can approximate any desired sojourn time distribution. Particular phase-type distributions include mixtures and convolutions of geometric distributions. The parameter estimation algorithm requires causal recursive estimation of the relevant statistics in each EM step. These statistics include the number of jumps of the bivariate Markov chain from one state to another, including self transitions, in the given observation interval, and first- and second-order statistical averages of the observable process in each state when the memoryless channel is Gaussian. We develop the explicit recursions and demonstrate the performance of the algorithm in estimating the model's parameter and its sojourn time distribution in a numerical example.
\end{abstract}

Index Terms-Markov processes, recursive estimation.

\section{INTRODUCTION}

$\mathbf{R}$ ECURSIVE parameter estimation is of great practical and theoretical importance since it enables online update of the parameter estimate as new data becomes available. A recursive variant of the popular EM approach was first proposed in [17], and then in [38] for estimating the parameter of a hidden Markov model (HMM). In this approach, estimates of the parameter and corresponding relevant statistics are updated in turn at each data point. By relevant statistics we mean the function or functions of the raw data which are needed in estimating the parameter of the model. For example, the relevant statistics for estimating the transition matrix of the underlying Markov chain in an HMM, is given by the number of jumps from each state to any other state, including self transitions, in the given observation interval. The estimates of the relevant

Manuscript received October 21, 2014; revised February 09, 2015; accepted February 10, 2015. Date of publication March 04, 2015; date of current version March 16, 2015. The associate editor coordinating the review of this manuscript and approving it for publication was Prof. Subhrakanti Dey. This work was supported in part by the U.S. National Science Foundation under Grant CCF0916568. (Corresponding Author: B. L. Mark.)

The authors are with the Department of Electrical and Computer Engineering, George Mason University, Fairfax, VA 22030 USA (e-mail: yephraim@gmu. edu; bmark@gmu.edu).

Color versions of one or more of the figures in this paper are available online at http://ieeexplore.ieee.org.

Digital Object Identifier 10.1109/TSP.2015.2408557 statistics are updated based on the last parameter estimate and the new observation, and the parameter estimate is updated based on the newly estimated statistics in a manner similar to that used by the EM algorithm. The approach was applied in [38] to finite-alphabet HMMs and its empirical convergence was demonstrated. It was pointed out in [38] that the approach is applicable to HMMs with continuous alphabets, for example, to HMMs with conditionally normal distributions. A similar approach was proposed about a decade later for finite-alphabet HMMs in [30], and subsequently for continuous alphabet in [8].

In this paper we adopt the approach of [38] and apply it to a discrete-time hidden bivariate Markov chain. This is a discretetime bivariate Markov chain observed through a discrete-time memoryless channel. For comparison, an HMM is a discretetime univariate Markov chain observed through a discrete-time memoryless channel [15, Section IV-A]. A bivariate Markov chain comprises a pair of random processes which are jointly, but not necessarily individually, Markov. Necessary and sufficient conditions for each of the two processes of the bivariate Markov chain to be Markov were given in [22, Theorem 6.3.2] for discrete-time Markov chains, and in [2, Theorem 3.1] for continuous-time Markov chains. In this paper, at least one of the two processes comprising the bivariate Markov chain is not Markov. The sojourn time of the bivariate Markov chain in each pair of joint states has a geometric distribution, just like in any univariate Markov chain. On the other hand, the sojourn time of the non-Markovian process of the bivariate Markov chain in each of its states has a discrete phase-type distribution [14, Eq. 8.7]. Thus, if the observable process of the hidden bivariate Markov chain is made dependent only on the non-Markovian process of the bivariate Markov chain, then the sojourn time distribution of the observable process in each of the dependable states is also phase-type. In HMMs, the distribution of the sojourn time of the observable process in each hidden state is geometric. Discrete, as well as continuous, phase-type distributions were introduced by Neuts [31]. The family of discrete phase-type distributions is very rich, and it contains the distributions of sums of independent geometric random variables, and mixtures of geometric distributions, as particular cases.

The hidden bivariate Markov chain model is a hidden semi-Markov process. An excellent reference on semi-Markov chains is [9, Chap. 10]. Converting an HMM into a hidden semi-Markov process with more favorable sojourn time distribution than the geometric distribution, was studied as early as 1980 in the pioneering work of Ferguson [18] in the context of speech recognition. In that approach, the HMM is supplemented with an explicit durational model which characterizes 
the distribution of the sojourn time of the observable process in each of the hidden states. Many papers have followed the work in [18], see, e.g., [20], [41], the survey of hidden semi-Markov processes with explicit durational model in [40], and the text [3]. The hidden bivariate Markov chain offers a natural structure without the need to specify an explicit distribution for the sojourn time. The most desirable distribution for a particular application is often unknown. Furthermore, estimation of the parameter of the hidden bivariate Markov chain is significantly simpler than that of the hidden semi-Markov process with explicit durational model. The hidden bivariate Markov chain was recently applied to cognitive radio spectrum sensing using batch EM parameter estimation in [32], and Rydén's [37] recursive parameter estimator in [39]. For cognitive radio spectrum sensing, characterization of the non-geometric sojourn time of the process in each state is of crucial importance. Another approach, called "iterative conditional estimation," was proposed for batch estimation of the parameter of a hidden semi-Markov process with non-explicit durational model in the form of a trivariate Markov chain [25].

Multivariate Markov chains have been studied over many years, and their established properties have been applied in numerous application areas. For example, in 1966 Baum and Petrie introduced a bivariate Markov chain in their analysis of the hidden Markov model [4]. In 1973 Rudemo defined a bivariate Markov chain and discussed its relation to an aggregating function of a continuous-time Markov chain [35]. The underlying standard state space model developed in the 1960s and used in Kalman filtering, is a multivariate Markov process. The observable process in that model is a hidden multivariate Markov process. Other examples of multivariate Markov processes include Itô processes; Markov modulated Poisson processes; Markov modulated Markov processes; batch Markovian arrival processes; aggregating functions of Markov chains, and variations of hidden Markov processes, see, e.g., [12]. A survey of these processes and their parameter estimation may be found in [14]. Most of these continuous-time models have discrete-time counterparts. Lumpability properties of discrete-time Markov chains were developed in [22], and of continuous-time multivariate Markov chains in [2]. Central limit theorems for multivariate semi-Markov sequences were developed in [1]. More recently, bivariate and trivariate Markov chains were studied and applied in a series of papers, utilizing the non-Markovian nature of the process components, see, e.g., [10], [24], [25], [34], and the references therein. These models were referred to as pairwise and triplet Markov chains, respectively.

A key to the recursive parameter estimation approach of [38], is the development of causal recursive estimators of the relevant statistics of the model. In this paper we develop such estimators for the discrete-time hidden bivariate Markov chain, and for the discrete-time bivariate Markov chain. These recursions apply to continuous and finite-alphabet HMMs, respectively. We study the recursions numerically and make a comparison with the batch EM algorithm. Analysis of the recursive parameter estimation algorithm, and any asymptotic performance results, are rather difficult to obtain due to the adaptive nature of the algorithm [7]. The numerical study is interesting and provides some insight into the workings of this algorithm.

For completeness, we mention the work of Holst and Lindgren [21] and Rydén [37] on recursive parameter estimation for HMMs using stochastic approximation procedures, and the work of LeGland and Mevel [27] on recursive maximum likelihood parameter estimation for HMMs. Asymptotic properties of the first two algorithms were established in [37], and of the third algorithm in [27]. All three algorithms require projection of the recursively estimated parameter onto the parameter space at the end of each iteration. Such projection is not required in the recursive EM-based procedure considered here.

The plan for this paper is as follows. In Section II we present the discrete-time hidden bivariate Markov chain. In Section III we outline the recursive parameter estimation approach for the hidden bivariate Markov chain. In Section IV we derive the causal recursions for the relevant statistics of the model. In Section V we present the results of a numerical study. In Section VI we provide some concluding remarks.

\section{Hidden Bivariate Markov Chain}

In this section we define the hidden bivariate Markov chain and present some of its relevant properties. Throughout this paper, we denote random variables by capital letters, and realizations of random variables by lowercase letters. We also denote a sequence of random variables, say $\left\{X_{0}, X_{1}, \ldots, X_{k}\right\}$, by $X_{0}^{k}$. The parameter of the hidden bivariate Markov chain is denoted by $\phi$. This parameter will be defined shortly. We use $P_{\phi}$ to denote a probability measure, and $p_{\phi}$ to denote a density of $P_{\phi}$.

\section{A. The Model}

Let $Z=\left\{Z_{t}, t=0,1,2, \ldots\right\}$ denote a discrete-time finite-state homogeneous bivariate Markov chain. This process comprises a pair of random processes, say $Z=(X, S)$, which are jointly Markov. The individual chains $X$ and $S$ need not be Markov, and we assume that the $X$ process is not Markov. The state space of $X$ is denoted by $\mathbb{X}$, and the state space of $S$ is denoted by $\mathbb{S}$. The state space of $Z$ is given by $\mathbb{Z}=\mathbb{X} \times \mathbb{S}$. We assume without loss of generality that $\mathbb{X}=\{1, \ldots, d\}$ and $\mathbb{S}=\{1, \ldots, r\}$ for some finite $d$ and $r$. Furthermore, we assume that the state pairs $\{(a, i) \in \mathbb{Z}\}$ are ordered lexicographically, and we denote the transition probability of $Z$ by

$$
h_{a b}(i j)=P_{\phi}\left(Z_{t}=(b, j) \mid Z_{t-1}=(a, i)\right) .
$$

The transition matrix $H=\left\{h_{a b}(i j)\right\}$ is conveniently written as a block matrix $H=\left\{H_{a b} ; a, b \in \mathbb{X}\right\}$, where $H_{a b}=\left\{h_{a b}(i j) ; i, j \in \mathbb{S}\right\}$ is an $r \times r$ matrix. The $S$-chain is Markov if and only if for every $a \in \mathbb{X}, \sum_{b \in \mathbb{X}} H_{a b}$ is independent of $a$ [22, Theorem 6.3.2]. A similar condition can be stated for the $X$-chain to be Markov upon re-partitioning of the transition matrix $H$. When $H$ is irreducible, it has a unique stationary distribution $\pi$ satisfying $\pi=\pi H$ [19, Theorem 6.9.21]. The process $\left\{Z_{t}\right\}$ is stationary if and only if $P_{\phi}\left(Z_{0}=(a, i)\right)=\pi_{a i}$ for all $(a, i) \in \mathbb{Z}$. We denote by $\zeta$ any initial distribution of $Z$, not necessarily the stationary distribution. The element of $\zeta$ 
that corresponds to state $(a, i)$ is denoted by $\zeta_{a i}$. We also use $\zeta_{a}=\left(\zeta_{a 1}, \ldots, \zeta_{a r}\right)$.

Statistical properties of discrete-time bivariate Markov chains were detailed in [14], [32, Chapter 8]. In particular, it was shown that the sojourn time of the non-Markovian process $X$ in each state $a \in \mathbb{X}$ has a discrete-time phase-type distribution with probability mass function ( $\mathrm{pmf}$ ) given by

$$
p_{\phi}(l \mid a)=\bar{\zeta}_{a} H_{a a}^{l-1}\left(I-H_{a a}\right) 1, \quad l=1,2, \ldots
$$

where 1 denotes a column vector of given dimension where all of its entries are ones, and $\bar{\zeta}_{a}=\zeta_{a} / \zeta_{a} 1$. The parameter of this pmf is $\left(\bar{\zeta}_{a}, H_{a a}\right)$ [31, p. 46]. The family of phase-type distributions is dense in the set of distributions on $\{0,1,2, \ldots\}$ $[26$, p. 54]. In discrete time, it contains the distributions of sums of independent geometric random variables as well as mixtures of geometric distributions.

Suppose now that $Z$ is observed through a memoryless channel whose output is given by $Y=\left\{Y_{t}, t=0,1,2, \ldots\right\}$. The joint density of the input and output of the channel is assumed to satisfy the following relations:

$$
\begin{aligned}
p_{\phi}\left(y_{0}^{n}, z_{0}^{n}\right) & =p_{\phi}\left(y_{0}, z_{0}\right) \prod_{t=1}^{n} p_{\phi}\left(y_{t}, z_{t} \mid y_{t-1}, z_{t-1}\right) \\
& =p_{\phi}\left(y_{0}, z_{0}\right) \prod_{t=1}^{n} p_{\phi}\left(y_{t}, z_{t} \mid z_{t-1}\right)
\end{aligned}
$$

for any positive integer $n$. Thus, the process $(Y, Z)$ is assumed Markov, and for each $t,\left(Y_{t}, Z_{t}\right)$ is conditionally independent of $Y_{t-1}$ given $Z_{t-1}$.

The model described so far is consistent with the standard HMM since it constitutes a Markov chain observed through a memoryless channel, see, e.g., [15, Eq. 4.1]. The transition density of the channel is given by $p_{\phi}\left(y_{t} \mid z_{t}, z_{t-1}\right)$. The facts that the Markov chain is bivariate rather than univariate, and that $Y_{t}$ depends on the pair of states $\left(Z_{t}, Z_{t-1}\right)$ rather than on $Z_{t}$ alone, are immaterial, since the standard HMM can be obtained by renaming the states $\{(a, i)\}$ in a larger state space. For this reason, we shall assume without loss of generality that $p_{\phi}\left(y_{t} \mid z_{t}, z_{t-1}\right)=p_{\phi}\left(y_{t} \mid z_{t}\right)$. It is well-known that the sojourn time of the observable process $Y$ of an HMM in each state of $Z$ is geometrically distributed, with parameter that depends on the underlying state. The geometric sojourn time distribution was found to be inadequate in certain applications as explained in Section I.

In the hidden bivariate Markov chain studied in this paper, we assume that for each time instant $t, Y_{t}$ is independent of $S_{t}$ given $X_{t}$. That is, $p_{\phi}\left(y_{t} \mid z_{t}\right)=p_{\phi}\left(y_{t} \mid x_{t}\right)$. In this model, the observable process $Y$ inherits its sojourn time from the non-Markovian process $X$, rather than from the Markovian process $Z$, and hence, the sojourn time distribution of $Y$ in each state of $X$ is discrete phase-type rather than geometric [14], [32, Eq. 8.7]. The algorithm for batch maximum likelihood estimation of the parameter of the hidden bivariate Markov chain is essentially the Baum algorithm for estimating the parameter of an HMM [32]. Hence, the proposed hidden bivariate Markov chain provides an elegant alternative to the HMM with more favorable and far more versatile sojourn time distributions [16], [32]. In this paper we focus on sequential parameter estimation for the hidden bivariate Markov chain and for the HMM. Parameter estimation for the two models can be discussed simultaneously as we shall see in the subsequent sections.

\section{B. Likelihood of Observable Process}

Define the conditional distribution

$$
F_{i j}^{a b}(y):=P_{\phi}\left(Y_{t} \leq y_{t}, Z_{t}=(b, j) \mid Z_{t-1}=(a, i)\right)
$$

and the corresponding transition density

$$
f_{i j}^{a b}\left(y_{t}\right)=\frac{\partial}{\partial y_{t}} F_{i j}^{a b}\left(y_{t}\right)=p_{\phi}\left(y_{t}, z_{t}=(b, j) \mid z_{t-1}=(a, i)\right) \text {. }
$$

Recall that the states $(X, S)$ are ordered lexicographically. Define the $d r \times d r$ transition density matrix by

$$
f\left(y_{t}\right)=\left\{f_{i j}^{a b}\left(y_{t}\right) ;(a, i),(b, j) \in \mathbb{Z}\right\} .
$$

The transition density matrix for the HMM version of our model, i.e., when $Y_{t}$ depends on $Z_{t}$, is given in terms of the transition matrix $H$ from (1) and the density which corresponds to $P_{\phi}\left(Y_{t} \leq y_{t} \mid Z_{t}=(b, j)\right)$. We denote that density by $g_{\theta_{b j}}\left(y_{t}\right)$ where $\theta_{b j}$ is its parameter. When this density is normal, then, $\theta_{b j}=\left(\mu_{b j}, \sigma_{b j}^{2}\right)$, where $\mu_{b j}$ denotes the mean and $\sigma_{b j}^{2}$ denotes the variance. For the hidden bivariate Markov chain version of our model, i.e., when $Y_{t}$ given $X_{t}$ is independent of $S_{t}$, the density $g_{\theta_{b j}}\left(y_{t}\right)$ is independent of $j$ and is given by $g_{\theta_{b}}\left(y_{t}\right)$. In either case,

$$
f\left(y_{t}\right)=H G\left(y_{t}\right)
$$

where for the HMM version,

$$
G\left(y_{t}\right)=\operatorname{diag}\left(g_{\theta_{b j}}\left(y_{t}\right),(b, j) \in \mathbb{Z}\right),
$$

and for the hidden bivariate Markov chain version,

$$
G\left(y_{t}\right)=\operatorname{diag}\left(g_{\theta_{b}}\left(y_{t}\right) I, b=1, \ldots, d\right)
$$

where $I$ is an $r \times r$ identity matrix. Define the $1 \times d r$ row vector $\nu_{y_{0}}=\left\{p_{\phi}\left(y_{0}, z_{0}\right) ; z_{0} \in \mathbb{Z}\right\}$. Then, the likelihood function of the hidden bivariate Markov chain is given by

$$
p_{\phi}\left(y_{0}^{n}\right)=\nu_{y_{0}} \prod_{t=1}^{n} f\left(y_{t}\right) \mathbf{1}
$$

We do not necessarily assume that the marginal distribution $\left\{p_{\phi}\left(z_{0}\right) ; z_{0} \in \mathbb{Z}\right\}$ of $\nu_{y_{0}}$ corresponds to the stationary distribution of $Z$. The parameter $\phi$ of our model comprises the initial distribution $\zeta$, the independent entries of $H$, and $\left\{\theta_{b j},(b, j) \in\right.$ $\mathbb{Z}\}$ for the HMM and $\left\{\theta_{b}, b \in \mathbb{X}\right\}$ for the hidden bivariate Markov chain.

\section{Forward Matrix Recursions}

The recursive estimators derived in this paper are conveniently expressed in terms of

$$
R(k, m):=\prod_{t=k}^{m} f\left(y_{t}\right)
$$


where $1 \leq k \leq m \leq n$. For a fixed $k$, we have

$$
\begin{aligned}
R(m, m-1) & :=I \\
R(k, m) & =R(k, m-1) f\left(y_{m}\right)
\end{aligned}
$$

where $I$ is an identity matrix. For $m=1,2, \ldots, n$, define $L(m)=\nu_{y_{0}} R(1, m)$, and let $L(0)=\nu_{y_{0}}$. We have,

$$
L(m)=L(m-1) f\left(y_{m}\right) .
$$

Note that for a given parameter $\phi$ and observation sequence $Y_{0}^{n}=y_{0}^{n}$, the $((a, i),(b, j))$ element of $R(k, m)$ is given by

$$
R_{a i, b j}(k, m)=p_{\phi}\left(y_{k}^{m}, z_{m}=(b, j) \mid z_{k-1}=(a, i)\right),
$$

and the $(b, j)$ element of $L(m)$ is given by

$$
L_{b j}(m)=p_{\phi}\left(y_{0}^{m}, z_{m}=(b, j)\right) .
$$

Numerical stability of the recursions in (12) and (13) is improved when scaling is introduced in each iteration. It is instructive to start with the description of the scaled version of $L(m)$, which we denote by $\tilde{L}(m)$. Let $c_{0}=\nu_{y_{0}} 1$, and let $\tilde{L}(0)=\nu_{y_{0}} / c_{0}$. The scaled version of (13) is given by

$$
\tilde{L}(m)=\frac{1}{c_{m}} \tilde{L}(m-1) f\left(y_{m}\right)
$$

where

$$
c_{m}=\tilde{L}(m-1) f\left(y_{m}\right) \mathbf{1}
$$

It follows that

$$
\tilde{L}(m)=\frac{1}{\prod_{t=0}^{m} c_{t}} L(m)
$$

$p_{\phi}\left(y_{0}^{m}\right)=\prod_{t=0}^{m} c_{t}, c_{t}=p_{\phi}\left(y_{t} \mid y_{0}^{t-1}\right)$ for $t \geq 1$, and the $(b, j)$ component of $\tilde{L}(m)$ is given by $\tilde{L}_{b j}(m)=P_{\phi}\left(Z_{m}=(b, j) \mid\right.$ $\left.y_{0}^{m}\right)$. The scaled version of $R(k, m)$ is given by

$$
\begin{aligned}
\tilde{R}(k, m) & =\frac{1}{\prod_{t=k}^{m} c_{t}} R(k, m) \\
& =\tilde{R}(k, m-1) \frac{f\left(y_{m}\right)}{c_{m}} .
\end{aligned}
$$

where $c_{m}$ is given in (17). The recursion $\tilde{R}(k, m)$ does not enjoy an appealing probabilistic interpretation as $\tilde{L}(m)$.

Clearly, $L$ and $R$ are reminiscent of the forward and backward densities of the HMM, respectively. The difference here is that for a fixed $k, R(k, m)$ is amenable to a forward recursion in $m$, while in the theory of HMMs, a fixed $m=n$ is used when considering an observation sequence $y^{n}$, and a backward recursion in $k$ is utilized. The idea of using $R$, as defined, in a forward recursive mode is due to Stiller and Radons [38].

\section{PARAMETER ESTIMATION APPROACH}

Our goal is to recursively estimate the parameter $\phi$ of the hidden bivariate Markov chain from a sequence of observations $y_{0}^{n}$. Consider first the batch EM algorithm, and assume that $\phi_{\kappa}$ is the parameter estimate at the end of the $\kappa$ th iteration. At the conclusion of the $\kappa+1$ st iteration, the new estimate of $\zeta_{b j}$ is given by

$$
\hat{\zeta}_{b j}(n)=P_{\phi_{\kappa}}\left(Z_{0}=(b, j) \mid y_{0}^{n}\right),
$$

and the new estimate of $h_{a b}(i j)$ is given by

$$
\hat{h}_{a b}(i j)=\frac{\hat{M}_{i j}^{a b}(n)}{\sum_{(\beta, l)} \hat{M}_{i l}^{a \beta}(n)}
$$

where $\hat{M}_{i j}^{a b}(n)$ denotes the conditional mean estimate given $y_{0}^{n}$ of the number of transitions of $Z$ from $(a, i)$ to $(b, j)$ in $[0, n]$. This number includes self transitions. Using the indicator function

$$
\varphi_{b j}(t)= \begin{cases}1, & Z_{t}=(b, j) \\ 0, & \text { otherwise }\end{cases}
$$

the number of transitions is given by

$$
M_{i j}^{a b}(n)=\sum_{t=1}^{n} \varphi_{a i}(t-1) \varphi_{b j}(t),
$$

and

$$
\begin{aligned}
\hat{M}_{i j}^{a b}(n) & =E_{\phi_{\kappa}}\left\{M_{i j}^{a b}(n) \mid y_{0}^{n}\right\} \\
& =\sum_{t=1}^{n} P_{\phi_{\kappa}}\left(Z_{t-1}=(a, i), Z_{t}=(b, j) \mid y_{0}^{n}\right) .
\end{aligned}
$$

For an HMM with normal densities $\left\{p_{\phi_{\kappa}}\left(y_{t} \mid z_{t}\right), z_{t} \in \mathbb{Z}\right\}$, with mean $\mu_{b j}$ and variance $\sigma_{b j}^{2}$ when $z_{t}=(b, j)$, define

$$
N_{j}^{b}(n ; \lambda)=\sum_{t=0}^{n} y_{t}^{\lambda} \varphi_{b j}(t)
$$

for $\lambda \in\{0,1,2\}$. Note, for example, that if the observations $\left\{y_{t}\right\}$ are clustered into the various states of the HMM, then $N_{j}^{b}(n ; 1)$ is the sum of the observations associated with state $(b, j)$. Let

$$
\begin{aligned}
\hat{N}_{j}^{b}(n ; \lambda) & =E_{\phi_{\kappa}}\left\{N_{j}^{b}(n ; \lambda) \mid y_{0}^{n}\right\} \\
& =\sum_{t=0}^{n} y_{t}^{\lambda} P_{\phi_{\kappa}}\left(Z_{t}=(b, j) \mid y_{0}^{n}\right) .
\end{aligned}
$$

The new estimates of the mean and variance at the conclusion of the $\kappa+1$ st iteration are, respectively, given by,

$$
\begin{aligned}
\hat{\mu}_{b j}(n) & =\frac{\hat{N}_{j}^{b}(n ; 1)}{\hat{N}_{j}^{b}(n ; 0)}, \\
\widehat{\sigma_{b j}^{2}}(n) & =\frac{\hat{N}_{j}^{b}(n ; 2)}{\hat{N}_{j}^{b}(n ; 0)}-\hat{\mu}_{b j}^{2}(n) .
\end{aligned}
$$

For a hidden bivariate Markov chain, $\mu_{b j}$ and $\sigma_{b j}^{2}$ are reduced to $\mu_{b}$ and $\sigma_{b}^{2}$, respectively. Thus, $P_{\phi_{\kappa}}\left(Z_{t}=(b, j) \mid y_{0}^{n}\right)$ in (27) and (28) should be substituted by $P_{\phi_{\kappa}}\left(X_{t}=b \mid y_{0}^{n}\right)$. It is well-known that the conditional probability in (24), and hence in (20) and (26), may be efficiently implemented using forward-backward recursions. 
In the EM approach, the entire data $y_{0}^{n}$ is used to update the estimate of the parameter in each iteration, and the value of the parameter remains fixed during each iteration. The value of the parameter may change only from one iteration to the next iteration. Here we adopt the approach of Stiller and Radons [38], in which the parameter $\phi$ is estimated recursively rather than iteratively. The recursive approach utilizes forward recursions for updating the estimates $\hat{\zeta}_{b j}(k)$ in (20), $\hat{M}_{i j}^{a b}(k)$ in (24), and $\hat{N}_{j}^{b}(n ; \lambda)$ in (26), for $k=1,2, \ldots$. These recursions are developed in the next section.

The recursive parameter estimation proceeds as follows. Suppose that at time $t=k$, a new observation $y_{k}$ becomes available while the current estimate of the parameter is $\phi_{k-1}$. Using $\left\{y_{k}, \phi_{k-1}\right\}$, a new parameter estimate $\phi_{k}$ is generated by recursively updating $\hat{\zeta}_{b j}(k), \hat{M}_{i j}^{a b}(k)$, and $\hat{N}_{j}^{b}(k ; \lambda)$, and substituting the updated $\hat{M}_{i j}^{a b}(k)$ and $\hat{N}_{j}^{b}(k ; \lambda)$ into (21), and (27) and (28), respectively. Note that if the estimates of the relevant statistics are recursively updated for $k=1, \ldots, n$ while the parameter is kept fixed at its initial value, and the parameter estimate is updated once only at $k=n$, then a single EM iteration results.

\section{Recursions For Hidden Bivariate Markov Chain}

In this section we develop the causal forward recursions required to implement the recursive parameter estimation approach. We develop the recursions for HMM with normal distributions, and comment on the modifications which are required in order to obtain the recursions for the hidden bivariate Markov chain model with normal distributions. We shall also develop causal forward recursions for the statistics of a bivariate Markov chain. To simplify notation, we shall refer to the current parameter estimate at $t=k$ as $\phi$ rather than $\phi_{k-1}$.

\section{A. Initial Distribution}

The density $p_{\phi}\left(z_{0}=(b, j), y_{0}^{k}\right)$, required for estimating $\zeta_{b j}$ in (20), can be obtained from $p_{\phi}\left(z_{0}=(b, j), z_{k}=(e, l), y_{0}^{k}\right)$ by summing over all $(e, l)$ in $\mathbb{Z}$. The inclusion of the additional condition of $Z_{k}=(e, l)$ is consistent with the state augmentation approach of Zeitouni and Dembo [42], and it facilitates the derivation of the desired forward recursions. The approach has been extensively used in the literature, see, e.g., [11], [13].

Let $\boldsymbol{\eta}_{b j}(k)$ denote a $1 \times d r$ row vector whose $(e, l)$ component is given by $p_{\phi}\left(z_{0}=(b, j), z_{k}=(e, l) \mid y_{0}^{k}\right)$. A recursion for $\boldsymbol{\eta}_{b j}(k)$ is obtained as follows. Let $\mathbf{1}_{b j}$ denote a $d r \times 1$ column vector with a one in the $(b, j)$ position and zeros elsewhere. Then,

$$
\begin{aligned}
& p_{\phi}\left(z_{0}=(b, j), z_{k}=(e, l), y_{0}^{k}\right) \\
& =\nu_{y_{0}} \mathbf{1}_{b j} \mathbf{1}_{b j}^{\prime} R(1, k) \mathbf{1}_{e l} \\
& =\nu_{y_{0}} \mathbf{1}_{b j} \mathbf{1}_{b j}^{\prime} R(1, k-1) f\left(y_{k}\right) \mathbf{1}_{e l}
\end{aligned}
$$

where ' denotes matrix transpose. Dividing both sides of (29) by $p\left(y_{0}^{k}\right)$, and using (19), we obtain,

$$
\begin{aligned}
p_{\phi}\left(z_{0}=(b, j), z_{k}=\right. & \left.(e, l) \mid y_{0}^{k}\right) \\
& =\frac{\nu_{y_{0}}}{c_{0}} \mathbf{1}_{b j} \mathbf{1}_{b j}^{\prime} \tilde{R}(1, k-1) \frac{f\left(y_{k}\right)}{c_{k}} \mathbf{1}_{e l} .
\end{aligned}
$$

Hence,

$$
\boldsymbol{\eta}_{b j}(k)=\boldsymbol{\eta}_{b j}(k-1) \frac{f\left(y_{k}\right)}{c_{k}},
$$

and the estimate of $\zeta_{b j}$ at time $k$ is given by

$$
\hat{\zeta}_{b j}(k)=\boldsymbol{\eta}_{b j}(k) \mathbf{1}
$$

The corresponding recursion for the hidden bivariate Markov chain follows when $f\left(y_{k}\right)$ is evaluated using (9) instead of (8).

\section{B. Transition Matrix}

A recursion for $\hat{M}_{i j}^{a b}(k)$ is obtained by utilizing the state augmentation approach of [42] as follows. Define a $1 \times d r$ row vector $\hat{\boldsymbol{M}}_{i j}^{a b}(k)$ whose $(e, l)$ component is given by

$$
\begin{aligned}
& \left\{\hat{\boldsymbol{M}}_{i j}^{a b}(k)\right\}_{e l} \\
& =\sum_{t=1}^{k} P_{\phi}\left(Z_{t-1}=(a, i), Z_{t}=(b, j), Z_{k}=(e, l) \mid y_{0}^{k}\right) .
\end{aligned}
$$

The estimate $\hat{M}_{i j}^{a b}(k)$ is given by

$$
\hat{M}_{i j}^{a b}(k)=\hat{M}_{i j}^{a b}(k) \mathbf{1},
$$

and from (21), the estimate of $h_{a b}(i j)$ at time $k$ is given by

$$
\hat{h}_{a b}^{k}(i j)=\frac{\hat{\boldsymbol{M}}_{i j}^{a b}(k) \mathbf{1}}{\sum_{(\beta, m)} \hat{\boldsymbol{M}}_{i m}^{a \beta}(k) \mathbf{1}} .
$$

The estimate $\left\{\hat{\boldsymbol{M}}_{i j}^{a b}(n)\right\}_{e l}$ is obtained by applying the Bayes rule to the summand of (33), and by using the Markov property of $(Y, Z)$. The derivation is similar to that given in [29] for a continuous-time bivariate Markov chain. We have

$$
\begin{aligned}
\left\{\hat{\boldsymbol{M}}_{i j}^{a b}(k)\right\}_{e l} & \sum_{t=1}^{k} \frac{p_{\phi}\left(z_{t-1}=(a, i), z_{t}=(b, j), z_{k}=(e, l), y_{0}^{k}\right)}{p_{\phi}\left(y_{0}^{k}\right)} \\
= & \sum_{t=1}^{k-1} \frac{p_{\phi}\left(y_{t+1}^{k}, z_{k}=(e, l) \mid z_{t}=(b, j)\right)}{p_{\phi}\left(y_{t+1}^{k} \mid y_{0}^{t}\right)} \\
& \cdot \frac{p_{\phi}\left(y_{t}, z_{t}=(b, j) \mid z_{t-1}=(a, i)\right)}{p_{\phi}\left(y_{t} \mid y_{0}^{t-1}\right)} \\
& \cdot \frac{p_{\phi}\left(y_{0}^{t-1}, z_{t-1}=(a, i)\right)}{p_{\phi}\left(y_{0}^{t-1}\right)} \\
& +\frac{p_{\phi}\left(y_{k}, z_{k}=(b, j) \mid z_{k-1}=(a, i)\right)}{p_{\phi}\left(y_{k} \mid y_{0}^{k-1}\right)} \\
& . \frac{p_{\phi}\left(y_{0}^{k-1}, z_{k-1}=(a, i)\right)}{p_{\phi}\left(y_{0}^{k-1}\right)} \mathbf{1}_{b j}^{\prime} \mathbf{1}_{e l} \\
= & \sum_{t=1}^{k} \frac{\nu_{y_{0}}}{c_{0}}\left[\prod_{m=1}^{t-1} \frac{f\left(y_{m}\right)}{c_{m}}\right] \mathbf{1}_{a i} \frac{g_{\theta_{b j}}\left(y_{t}\right) h_{a b}(i j)}{c_{t}} \\
& \cdot \mathbf{1}_{b j}^{\prime}\left[\prod_{m=t+1}^{k} \frac{f\left(y_{m}\right)}{c_{m}}\right] \mathbf{1}_{e l}
\end{aligned}
$$


where we have interpreted $\prod_{m=k+1}^{k} \frac{f\left(y_{m}\right)}{c_{m}}$ as being equal to $I$. Hence, for $k \geq 1$,

$$
\begin{aligned}
\hat{\boldsymbol{M}}_{i j}^{a b}(k)=\hat{\boldsymbol{M}}_{i j}^{a b}(k-1) \frac{f\left(y_{k}\right)}{c_{k}} & \\
& +\frac{g_{\theta_{b j}}\left(y_{k}\right) h_{a b}(i j)}{c_{k}} \tilde{L}(k-1) \mathbf{1}_{a i} \mathbf{1}_{b j}^{\prime},
\end{aligned}
$$

where $\hat{\boldsymbol{M}}_{i j}^{a b}(0)=0$, and $\tilde{L}(k-1)$ is calculated using (16). This is the recursion for the HMM. The corresponding recursion for the hidden bivariate Markov chain is obtained when $f\left(y_{k}\right)$ is evaluated using (9) instead of (8), and similarly, $g_{\theta_{b j}}\left(y_{k}\right)$ in (37) is replaced by $g_{\theta_{b}}\left(y_{k}\right)$ for all $j \in \mathbb{S}$.

\section{Mean and Variance of Normal Distribution}

Define the $1 \times d r$ row vector $\hat{\boldsymbol{N}}_{j}^{b}(k ; \lambda)$ whose $(e, l)$ element is given by

$$
\left\{\hat{N}_{j}^{b}(k ; \lambda)\right\}_{e l}=\sum_{t=0}^{k} y_{t}^{\lambda} P_{\phi}\left(Z_{t}=(b, j), Z_{k}=(e, l) \mid y_{0}^{k}\right) .
$$

Let $\hat{\mu}_{b j}(k)$ denote the estimate of $\mu_{b j}$ given $y_{0}^{k}$. From (27),

$$
\hat{\mu}_{b j}(k)=\frac{\hat{\boldsymbol{N}}_{j}^{b}(k ; 1) \mathbf{1}}{\hat{\boldsymbol{N}}_{j}^{b}(k ; 0) \mathbf{1}} .
$$

Let $\widehat{\sigma_{b j}^{2}}(k)$ denote the estimate of $\sigma_{b j}^{2}$ given $y_{0}^{k}$. From (28),

$$
\widehat{\sigma_{b j}^{2}}(k)=\frac{\hat{\boldsymbol{N}}_{j}^{b}(k ; 2) \mathbf{1}}{\hat{\boldsymbol{N}}_{j}^{b}(k ; 0) \mathbf{1}}-\hat{\mu}_{b j}^{2}(k) .
$$

A recursion for $\hat{\boldsymbol{N}}_{j}^{b}(k ; \lambda)$ follows from a procedure similar to that used in the derivation of (36), and from (7) and (16). We have for $k \geq 0$,

$$
\hat{\boldsymbol{N}}_{j}^{b}(k ; \lambda)=\hat{\boldsymbol{N}}_{j}^{b}(k-1 ; \lambda) \frac{f\left(y_{k}\right)}{c_{k}}+y_{k}^{\lambda} \tilde{L}(k) \mathbf{1}_{b j} \mathbf{1}_{b j}^{\prime}
$$

where $\hat{\boldsymbol{N}}_{j}^{b}(-1 ; \lambda):=0$.

For a hidden bivariate Markov chain, the recursion (41) should be summed over all $j \in \mathbb{S}$ and used in (39) and (40) to produce the estimates of $\mu_{b}$ and $\sigma_{b}^{2}$, respectively. In evaluating this recursion, (9) instead of (8) should be used, and similarly, $g_{\theta_{b}}\left(y_{k}\right)$ should be used instead of $g_{\theta_{b j}}\left(y_{k}\right)$ for all $j \in \mathbb{S}$.

\section{Recursive Auxiliary Function}

The recursive algorithm described above may also be derived from maximization of a recursive version of the EM auxiliary function. That function is given by

$$
Q(k)=E_{\phi^{\dagger}}\left\{\log p_{\phi}\left(Z_{0}^{k}, y_{0}^{k}\right) \mid y_{0}^{k}\right\}
$$

where $\phi^{\dagger}$ is the assumed known parameter at a given EM iteration. Excluding estimation of the initial distribution, the aux- iliary function for estimating $\phi=\left\{h_{a b}(i j), g_{\theta_{b j}}\right\}$ can be obtained from summation of

$$
\begin{array}{r}
\left\{\boldsymbol{Q}_{i j}^{a b}(k)\right\}_{e l}=\sum_{t=1}^{k} p_{\phi^{\dagger}}\left(z_{t-1}=(a, i), z_{t}=(b, j), z_{k}=(e, l) \mid y_{0}^{k}\right) \\
\cdot \log p_{\phi}\left(z_{t}=(b, j), y_{t} \mid z_{t-1}=(a, i)\right)
\end{array}
$$

over $\{(a, i),(b, j),(e, l)\}$. Following a procedure similar to that used in the derivation of (37), it can be shown that the $1 \times d r$ row vector $\boldsymbol{Q}_{i j}^{a b}(k)$, whose $(e, l)$ component is given in (43), satisfies the following recursion:

$$
\begin{aligned}
& \boldsymbol{Q}_{i j}^{a b}(k)=\boldsymbol{Q}_{i j}^{a b}(k-1) \frac{f^{\dagger}\left(y_{k}\right)}{c_{k}} \\
& +\frac{g_{\theta_{b j}}^{\dagger}\left(y_{k}\right) h_{a b}^{\dagger}(i j)}{c_{k}} \tilde{L}(k-1) \mathbf{1}_{a i} \mathbf{1}_{b j}^{\prime} \log \left(g_{\theta_{b j}}\left(y_{k}\right) h_{a b}(i j)\right),
\end{aligned}
$$

where $\boldsymbol{Q}_{i j}^{a b}(k-1), \tilde{L}(k-1)$, and $c_{k}$, are calculated based on $\phi^{\dagger}$. Maximization of $\boldsymbol{Q}_{i j}^{a b}(k)$ over $\phi=\left\{h_{a b}(i j), g_{\theta_{b j}}\right\}$, subject to suitable constraints, results in the EM re-estimation formulas in (35), (39), and (40) which incorporate the recursions for $\hat{\boldsymbol{M}}_{i j}^{a b}(n)$ and $\hat{\boldsymbol{N}}_{j}^{b}(k ; \lambda)$ from (37) and (41), respectively. Unfortunately, a true EM recursion is not possible with this procedure, since $\phi^{\dagger}$ in an EM recursion must remain fixed for all $k$ 's, while here $\phi^{\dagger}$ is taken to be the last parameter estimate corresponding to time $k-1$.

Recursive estimation of the auxiliary function for independent identically distributed data from the exponential family, using stochastic approximation, was studied in [7].

\section{E. Exponential Forgetting}

The recursions for $\hat{\boldsymbol{M}}_{i j}^{a b}(k)$ and $\hat{\boldsymbol{N}}_{j}^{b}(k ; \lambda)$, may be heuristically modified to emphasize the probability of events at time instants closer to $k$ than at earlier time instants. This is done here, similarly to [38], by introducing an exponential forgetting factor. We demonstrate this approach for $\hat{\boldsymbol{M}}_{i j}^{a b}(k)$ only. For $0<\rho<1$ and a given parameter $\phi=\phi_{k-1}$, the modified $\left\{\hat{\boldsymbol{M}}_{i j}^{a b}(k)\right\}_{e l}$ is given by

$$
\begin{aligned}
& \left\{\hat{\boldsymbol{M}}_{i j}^{a b}(k)\right\}_{e l} \\
& =\sum_{t=1}^{k} \rho^{k-t} \frac{p_{\phi}\left(z_{t-1}=(a, i), z_{t}=(b, j), z_{k}=(e, l), y_{0}^{k}\right)}{p_{\phi}\left(y_{0}^{k}\right)} .
\end{aligned}
$$

The resulting recursion is given by

$$
\begin{aligned}
& \hat{\boldsymbol{M}}_{i j}^{a b}(k) \\
& =\rho \hat{\boldsymbol{M}}_{i j}^{a b}(k-1) \frac{f\left(y_{k}\right)}{c_{k}}+\frac{g_{\theta_{b j}}\left(y_{k}\right) h_{a b}(i j)}{c_{k}} \tilde{L}(k-1) \mathbf{1}_{a i} \mathbf{1}_{b j}^{\prime} .
\end{aligned}
$$

Moreover, by summing (45) over all $\{(a, i),(b, j)\}$ we obtain

$$
\tilde{L}(k-1)=\frac{1}{\sum_{t=1}^{k-1} \rho^{k-1-t}} \sum_{a^{\prime}, i^{\prime}} \sum_{b^{\prime}, j^{\prime}} \hat{\boldsymbol{M}}_{i^{\prime} j^{\prime}}^{a^{\prime} b^{\prime}}(k-1),
$$


which can be used in (46). Other forgetting factors besides the exponential one used here are possible.

Note that in classical recursive estimation, the exponential forgetting factor is applied to the error criterion, e.g., to the sum of squared errors in weighted least squares, while here it was applied to an estimate of a statistic from which the desired parameter is estimated. This is due to the use of the maximum likelihood criterion which does not involve an explicit error term.

\section{F. Recursions for Bivariate Markov Chains}

In this section we provide recursions for estimating the initial distribution and the transition matrix of a bivariate Markov chain. For this model, $X$ is the observable process and $S$ is the underlying process. This model generalizes finite-alphabet HMMs. Forward matrix recursions similar to those given in Section II-C can be defined here, with $f_{i j}^{a b}\left(y_{t}\right)$ in (5) replaced by $h_{a b}(i j)$, and $f\left(y_{t}\right)$ in (6) replaced by $H_{x_{t-1}, x_{t}}$.

The recursion for estimating the initial distribution of $S_{0}$ given $x_{0}^{k}$ can be derived similarly to (31) and (32). Let $\nu_{x_{0}}=\left\{p_{\phi}\left(x_{0}, s_{0}\right) ; s_{0} \in \mathbb{S}\right\}$, and let $\boldsymbol{\eta}_{j}(k)$ denote the $1 \times r$ row vector whose $l$ th component is given by $p_{\phi}\left(s_{0}=j, s_{k}=l \mid x_{0}^{k}\right)$. Then,

$$
\boldsymbol{\eta}_{j}(k)=\boldsymbol{\eta}_{j}(k-1) \frac{H_{x_{k-1}, x_{k}}}{c_{k}},
$$

from which the desired estimate is obtained as $\boldsymbol{\eta}_{j}(k) \mathbf{1}$. The transition matrix estimate is obtained from (35) where the $l$ th component of the vector $\hat{M}_{i j}^{a b}(k)$ is defined by

$$
\begin{aligned}
& \left\{\hat{\boldsymbol{M}}_{i j}^{a b}(k)\right\}_{l} \\
& =\sum_{\substack{t=1: x_{t-1}=a \\
x_{t}=b}}^{k} P_{\phi}\left(Z_{t-1}=(a, i), Z_{t}=(b, j), S_{k}=l \mid x_{0}^{k}\right) .
\end{aligned}
$$

Following a procedure similar to that used in the derivation of (37), it can be shown that the vector $\hat{M}_{i j}^{a b}(k)$ satisfies the following recursion:

$$
\begin{aligned}
\hat{\boldsymbol{M}}_{i j}^{a b}(k) & =\hat{\boldsymbol{M}}_{i j}^{a b}(k-1) \frac{H_{x_{k-1}, x_{k}}}{c_{k}} \\
& +I\left(X_{k-1}=a, X_{k}=b\right) \frac{h_{a b}(i j)}{c_{k}} \tilde{L}(k-1) \mathbf{1}_{i} \mathbf{1}_{j}^{\prime} .
\end{aligned}
$$

\section{Numerical EXAMPLE}

In this section, we illustrate the proposed causal recursive parameter estimation approach with a simple numerical example. We have used a bivariate Markov chain with order $d=r=2$ and transition matrix given by

$$
H=\left[\begin{array}{l|l}
H_{11} & H_{12} \\
\hline H_{21} & H_{22}
\end{array}\right]=\left[\begin{array}{ll|ll}
0.25 & 0.25 & 0.25 & 0.25 \\
0.8 & 0.1 & 0 & 0.1 \\
\hline 0.3 & 0 & 0.3 & 0.4 \\
0.1 & 0.6 & 0.1 & 0.2
\end{array}\right] .
$$

Note that $H_{11}+H_{12} \neq H_{21}+H_{22}$ and hence the $S$-chain of the bivariate Markov chain is not Markov. It can be shown similarly, by re-partitioning $H$, that the $X$-chain is also not Markov. The bivariate Markov chain was assumed to be observed through a white Gaussian channel. The vector of means and the vector of variances of the two conditionally Gaussian densities of the channel are, respectively, given by

$$
\mu=[-10,10], \quad \sigma^{2}=[5,1] .
$$

We denote this true parameter of the hidden bivariate Markov chain by $\phi^{0}$. We have tried two different initial distributions which will be specified shortly.

The process and estimators were implemented in Python using the NumPy library. We used the value $\rho=0.9999$ for the exponential forgetting factor. In practice, a warmup period is needed in order to obtain meaningful values for the statistics $\hat{\boldsymbol{M}}_{i j}^{a b}(k)$ and $\hat{\boldsymbol{N}}_{j}^{b}(k ; \lambda)$. During that period, only these estimators are updated while the initial parameter estimate remains fixed. Following the warmup period, the alternate estimation procedure described in Section III is followed, where the parameter and statistics estimates are updated for each new observation. In our numerical work, we have set the warmup period to the first 200 observations.

The first set of results is shown in Table I. Here, we have estimated the parameter of the hidden bivariate Markov chain, using the causal recursive approach of this paper, from a set of 50 sequences, each consisting of 100,000 observations, which were generated using the true parameter $\phi^{0}$. The initial parameter estimate is shown as $\phi_{0}$. The parameter estimates obtained from $\phi_{0}$ and the observation sequences are denoted by $\hat{\phi}_{\mathrm{c}}(1), \ldots, \hat{\phi}_{\mathrm{c}}(50)$. In the table, $\hat{E}\left[\hat{\phi}_{\mathrm{c}}\right]$ denotes the average of these estimates, and $\mathrm{MSE}\left[\hat{\phi}_{\mathrm{c}}\right]$ denotes the sample mean squared error (MSE), in estimating each component of $\phi^{0}$. The table also shows the sample mean, $\hat{E}\left[\hat{\phi}_{\text {nc }}\right]$, and the sample MSE, MSSE $\left[\hat{\phi}_{\mathrm{nc}}\right]$, in estimating each component of $\phi^{0}$ using the batch (or non-causal recursive) EM approach from Section III. This approach was applied to the first 2,000 observations from each sequence, and the EM was allowed to complete 50 iterations for each estimate. By using 2,000 data samples from each observation sequence in each of the $50 \mathrm{EM}$ iterations, we have effectively used the equivalent of the 100,000 samples employed by the causal estimation in each sequence. These results show that the accuracy of the causal estimates is similar to that of the non-causal estimates and even better for some of the parameter components. The estimates for the components of the parameter associated with the observable process, i.e., $\mu$ and $\sigma^{2}$, are very accurate, whereas those for the transition matrix of the underlying bivariate Markov chain are considerably less accurate.

Fig. 1 shows the transient behavior of the causal estimator for selected components of the transition matrix: $h_{11}(12), h_{12}(11)$, $h_{21}(22)$, and $h_{22}(21)$. During the first 200 time units, the causal parameter estimate is equal to the initial parameter. From Fig. 1, the estimates appear to change substantially until about $t=$ 2500 , after which the estimates change more slowly, appearing to converge by $t=4000$. Fig. 2 shows the transient behavior of the causal estimates for $\mu_{2}$ and $\sigma_{2}^{2}$. In contrast to the estimates for the transition matrix, the estimates for $\mu$ and $\sigma^{2}$ converge very quickly to their true values. 
TABLE I

Comparison of Causal and Noncausal Parameter Estimates With initial Parameter $\phi_{0}$ AND TRUe Parameter $\phi^{0}$ Given by (51)-(52)

\begin{tabular}{c||cc||cc|cc||cc|cc|}
\cline { 2 - 10 } \multicolumn{1}{c|}{} & \multicolumn{2}{c||}{$\phi_{0}$} & \multicolumn{2}{c|}{$\hat{E}\left[\hat{\phi}_{\mathrm{c}}\right]$} & \multicolumn{2}{c||}{$\mathrm{M} \hat{\mathrm{SE}}\left[\hat{\phi}_{\mathrm{c}}\right] \times 10^{3}$} & \multicolumn{2}{c|}{$\hat{E}\left[\hat{\phi}_{\mathrm{nc}}\right]$} & $\mathrm{MSE}\left[\hat{\phi}_{\mathrm{nc}}\right] \times 10^{3}$ \\
\hline \hline$H_{11}$ & 0.2 & 0.2 & 0.22 & 0.25 & 1.26 & 0.06 & 0.20 & 0.25 & 4.31 & 1.71 \\
& 0.7 & 0.2 & 0.72 & 0.20 & 6.36 & 9.82 & 0.74 & 0.18 & 6.92 & 8.52 \\
\hline$H_{12}$ & 0.2 & 0.3 & 0.24 & 0.30 & 0.18 & 2.52 & 0.24 & 0.31 & 0.47 & 4.2 \\
& 0 & 0.1 & 0 & 0.08 & 0 & 0.39 & 0 & 0.08 & 0 & 1.79 \\
\hline$H_{21}$ & 0.2 & 0 & 0.21 & 0 & 9.15 & 0 & 0.20 & 0 & 10.6 & 0 \\
& 0.2 & 0.5 & 0.16 & 0.57 & 4.12 & 0.83 & 0.16 & 0.58 & 3.94 & 1.32 \\
\hline$H_{22}$ & 0.4 & 0.4 & 0.29 & 0.51 & 0.29 & 12.2 & 0.29 & 0.50 & 1.64 & 11.8 \\
& 0.15 & 0.15 & 0.10 & 0.17 & 0.05 & 1.01 & 0.10 & 0.16 & 0.50 & 1.74 \\
\hline$\mu$ & -5 & 5 & -10.0 & 10.0 & 0.41 & 0.11 & -10.0 & 10.0 & 3.38 & 1.18 \\
\hline$\sigma^{2}$ & 6 & 2 & 5.00 & 1.00 & 4.07 & 0.25 & 5.02 & 0.99 & 47.2 & 2.55 \\
\hline
\end{tabular}

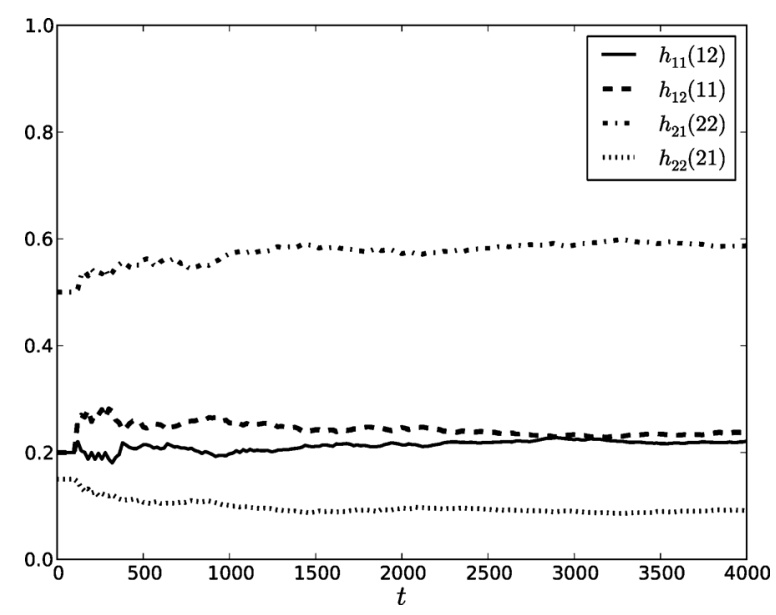

Fig. 1. Transient behavior of causal estimator for four components of the transition matrix $H$.

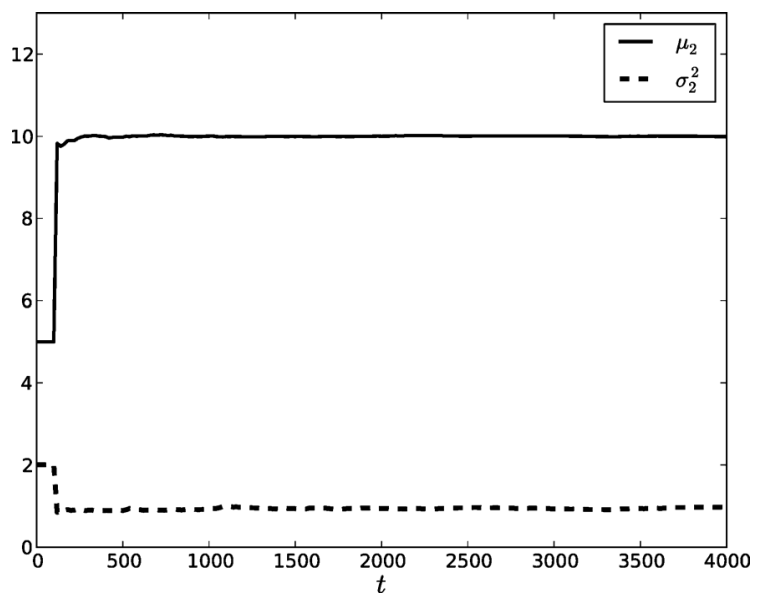

Fig. 2. Transient behavior of causal estimator for components of $\mu_{2}$ and $\sigma_{2}^{2}$.

The initial parameter $\phi_{0}$ used in Table I is relatively "close" to the true parameter $\phi^{0}$ given in (51)-(52). Table II compares the causal and non-causal parameter estimates for a different initial parameter $\phi_{0}$, which bears less resemblance to the true parameter $\phi^{0}$, except for the component $\mu$, which is slightly "closer" to the true value in this case. We see that the accuracy of the parameter estimates, both causal and non-causal, are significantly poorer here, with the exception of the observation parameter components $\mu$ and $\sigma^{2}$. Nevertheless, the accuracy of the causal and non-causal parameter estimates are again similar with respect to each other.

The estimates of the transition matrix obtained from the initial parameter shown in Table II resulted in relatively large bias and high MSE. It is interesting to note that these same estimates may still be useful by providing reasonably accurate representations of certain statistics of the hidden bivariate Markov chain, which are more relevant than the bias and MSE measures in a given application. Of particular interest is the representation of the parameter of the phase-type sojourn time distribution of the hidden bivariate Markov chain. Fig. 3 shows four phase-type pmfs for state 2 , as obtained from (2), using the true parameter, the initial parameter from Table II, and two causal parameter estimates obtained from two sequences of 100,000 and 200,000 samples, respectively. The phase-type pmfs corresponding to these two estimates are denoted by "est-100k" and "est-200k," respectively. Clearly, both parameter estimates improve the representation of the phase-type pmf of the bivariate Markov chain as obtained from the initial parameter, and the "est-200k" phase-type pmf estimate virtually coincides with the true phase-type pmf of the bivariate Markov chain.

Fig. 4 shows similar phase-type pmfs for state 1 as obtained from the initial parameter from Table II, the true parameter, and three causal parameter estimates obtained using three data sequences of increasing length. For this state, the true phase-type pmf does not resemble the geometric pmf as is the case with state 2 . The dashed curve labelled "est-100k" corresponds to the causal parameter estimate from Table II. Here, the "est-100k" curve is closer to the phase-type pmf associated with the initial parameter than that associated with the true parameter. When 200,000 observations were used, the causal parameter estimate yielded a phase-type pmf that is significantly closer to that for the true parameter. Increasing the number of observations to 400,000 , resulted in further, though marginal, improvement in the estimate of the phase-type pmf of state 1 .

Figs. 3 and 4 demonstrate that the phase-type pmfs associated with the causal parameter estimates could converge to the true phase-type pmfs, even when the parameter estimate itself does not converge to the true parameter. However, the results shown in Fig. 4 suggest that the convergence speed can be rather slow. It should be noted that the shape of the phase-type pmf can be quite sensitive to the true values of the components in the transition matrix; i.e., small changes in these values can result in significant changes to the shape of the phase-type pmf curve. 
TABLE II

Comparison of Causal and Non-Causal Estimates for ANother Initial Parameter $\phi_{0}$ and True Parameter $\phi^{0}$ Given by (51)-(52).

\begin{tabular}{c||cc||cc|cc||cc|cc|}
\cline { 2 - 10 } \multicolumn{1}{c|}{} & \multicolumn{2}{c||}{$\phi_{0}$} & \multicolumn{2}{c|}{$\hat{E}\left[\hat{\phi}_{\mathrm{c}}\right]$} & \multicolumn{2}{c||}{$\mathrm{M} \hat{\mathrm{SE}}\left[\hat{\phi}_{\mathrm{c}}\right] \times 10^{3}$} & $\hat{E}\left[\hat{\phi}_{\mathrm{nc}}\right]$ & \multicolumn{2}{c|}{$\mathrm{M} \hat{\mathrm{SE}}\left[\hat{\phi}_{\mathrm{nc}}\right] \times 10^{3}$} \\
\hline \hline$H_{11}$ & 0.6 & 0.1 & 0.60 & 0.14 & 123 & 12.4 & 0.58 & 0.21 & 110 & 3.12 \\
& 0.2 & 0.1 & 0.14 & 0.06 & 430 & 1.47 & 0.09 & 0.01 & 502 & 7.72 \\
\hline$H_{12}$ & 0.2 & 0.1 & 0.20 & 0.06 & 2.16 & 37.0 & 0.16 & 0.05 & 8.47 & 40.5 \\
& 0 & 0.7 & 0 & 0.79 & 0 & 481 & 0 & 0.00 & 0 & 631 \\
\hline$H_{21}$ & 0.6 & 0 & 0.78 & 0 & 226 & 0 & 0.67 & 0 & 143 & 0 \\
& 0.05 & 0.05 & 0.20 & 0.04 & 9.12 & 312 & 0.38 & 0.04 & 83 & 1.32 \\
\hline$H_{22}$ & 0.2 & 0.2 & 0.16 & 0.06 & 18.8 & 115 & 0.21 & 0.12 & 11.0 & 83.3 \\
& 0.25 & 0.65 & 0.40 & 0.36 & 89.6 & 26.9 & 0.25 & 0.32 & 26.9 & 19.2 \\
\hline$\mu$ & -4 & 7 & -10.0 & 10.0 & 0.00 & 0.00 & -10.0 & 10.0 & 3.38 & 1.18 \\
\hline$\sigma^{2}$ & 2 & 8 & 4.99 & 1.00 & 4.41 & 0.23 & 5.02 & 0.99 & 47.2 & 2.55 \\
\hline
\end{tabular}

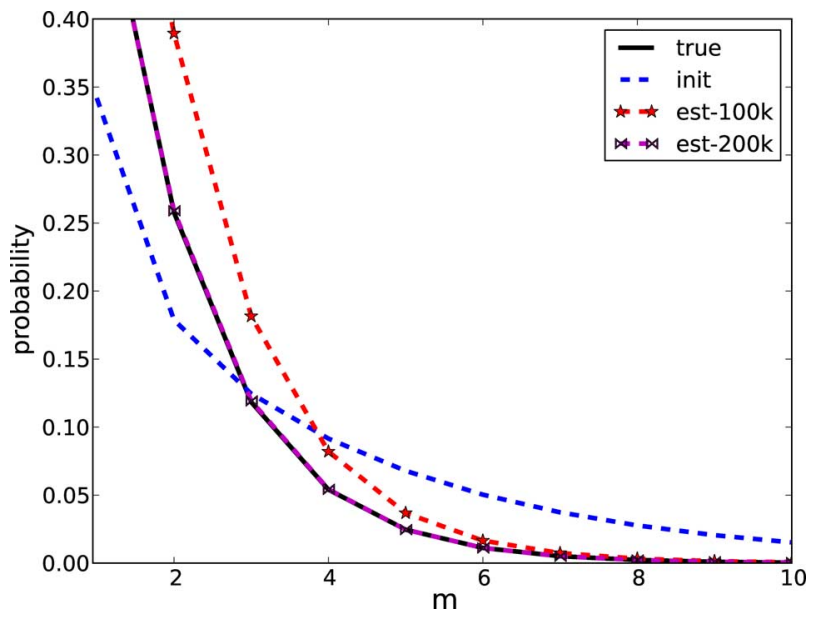

Fig. 3. State 2 sojourn time distributions for true parameter, initial parameter, and parameter estimates.

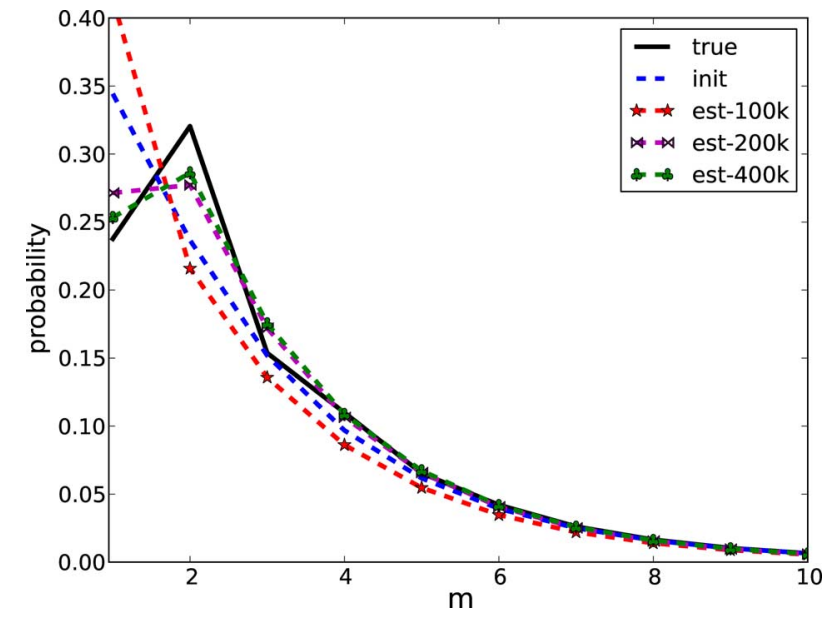

Fig. 4. State 1 sojourn time distributions for true parameter, initial parameter, and parameter estimates.

\section{COMMENTS}

We have developed an algorithm for recursive parameter estimation for a hidden bivariate Markov chain. The motivation for using this model lies in its significantly more general sojourn time distribution compared to that of the HMM. We have developed forward recursions for all statistics required by the EM-based recursive parameter estimation algorithm. Numeric results show that upon convergence, the quality of the estimates obtained using the recursive parameter estimator is similar to that obtained using the batch EM approach. If the maximum likelihood estimator is achieved by either procedure, then consistency and asymptotic normality of the estimator could be inferred from similar results developed for the HMM [5], [28]. We have used the bias and the mean squared error to evaluate and compare the various parameter estimates. Furthermore, we showed that the sojourn time distributions of the estimated models match quite closely those of the true model.

We note that the algorithm could also be used in a block mode. Here, the parameter estimate at the beginning of each data block is kept constant while the estimates of the relevant statistics are recursively updated for each data point in the block. Once the last data point in the block is processed, the parameter estimate is updated, and the processing of a new block commences in a similar manner. When the block size is one, we return to the original algorithm. In our study, we have experimented with several block sizes, but the block mode approach did not improve on the original scalar recursive approach described in this paper.

The model studied in this paper is a particular trivariate Markov process $(Y, X, S)=\left(\left\{Y_{t}\right\},\left\{X_{t}\right\},\left\{S_{t}\right\}\right)$ in which $(X, S)$ is a bivariate Markov chain, and $Y$ comprises a sequence of conditionally independent random variables given $(X, S)$. When $\left\{Y_{t}\right\}$ depends only on $\left\{X_{t}, S_{t}\right\}$, then the trivariate Markov process becomes a standard HMM, see, e.g., [15]. When $Y_{t}$ is independent of $S_{t}$ given $X_{t}$, and $X$ is not Markov, the trivariate Markov process becomes the hidden bivariate Markov chain of this paper. One could remove the assumption that $(X, S)$ is a bivariate Markov chain, and make $Y$ dependent on the pair $(X, S)$. In either of the last two models, $Y$ depends on a non-Markovian process, and its sojourn time in each state of the process it depends on, is phase-type. One could further generalize the process, and introduce dependence of $Y_{t}$ on previous samples of $Y$ as well as on $(X, S)$. The reader is referred to the work in [6], [10], [23]-[25], [33], [34] for possible extensions along these lines. A possible benefit of the latter model is that it can better capture the correlation of the process $Y$. This, however, could also be accomplished in our hidden bivariate Markov chain model, by allowing $Y$ to be a vector process where the components of each vector are correlated. Such model is significantly simpler than the general trivariate Markov process, and is likely to perform satisfactorily in most practical applications, since ignoring the correlation at 
the boundaries of the vectors of $Y$ cannot have any significant effects [36].

\section{ACKNOWLEDGMENT}

The authors thank the referees for their extensive comments which helped improve the presentation of this work.

\section{REFERENCES}

[1] F. Ball, "Central limit theorems for multivariate semi-Markov sequences and processes, with applications," J. Appl. Probab., vol. 36, no. 2, pp. 415-432, 1999.

[2] F. Ball and G. F. Yeo, "Lumpability and marginalisability for continuous time Markov chains," J. Appl. Probab., vol. 30, no. 3, pp. 518-528, 1993.

[3] V. S. Barbu and N. Limnios, Semi-Markov Chains and Hidden Semi-Markov Models Toward Applications: Their Use in Reliability and DNA Analysis, ser. Lecture Notes in Statistics. New York, NY, USA: Springer, 2008, vol. 191.

[4] L. E. Baum and T. Petrie, "Statistical inference for probabilistic functions of finite state Markov chains," Ann. Math. Statist., vol. 37, pp. 1554-1563, 1966.

[5] P. J. Bickel, Y. Ritov, and T. Rydén, "Asymptotic normality of the maximum-likelihood estimator for general hidden Markov models," Ann. Statist., vol. 26, no. 4, pp. 1614-1635, 1998.

[6] M. Y. Boudaren, E. Monfrini, and W. Pieczynski, "Unsupervised segmentation of random discrete data hidden with switching noise distributions," IEEE Signal Process. Lett., vol. 19, no. 10, pp. 619-622, 2012.

[7] O. Cappé and E. Moulines, "On-line expectation-maximization algorithm for latent data models," J. Roy. Statist. Soc.. Ser. B (Statist. Methodol.), vol. 71, no. 3, pp. 593-613, 2009.

[8] O. Cappé, "Online EM algorithm for hidden Markov models," J. Computat. Graphic. Statist., vol. 20, no. 3, pp. 728-749, 2011.

[9] E. Çinlar, Introduction to Stochastic Processes. Baltimore, MD, USA: The Johns Hopkins Univ. Press, 1981.

[10] S. Derrode and W. Pieczynski, "Signal and image segmentation using pairwise Markov chains," IEEE Trans. Signal Process., vol. 52, no. 9, pp. 2477-2489, 2004

[11] R. J. Elliott, L. Aggoun, and J. B. Moore, Hidden Markov Models: Estimation and Control. New York, NY, USA: Springer-Verlag, 1977.

[12] Y. Ephraim, "State dependent dynamical system model for speech signals," in Proc. Conf. Inf. Sci. Syst., Mar. 1992, pp. 595-600.

[13] Y. Ephraim and B. L. Mark, "Explicit forward recursive estimators for Markov modulated Markov processes," Stoch. Models, vol. 28, no. 3, pp. 359-387, 2012.

[14] Y. Ephraim and B. L. Mark, "Bivariate Markov processes and their estimation," Found. Trends Signal Process., vol. 6, no. 1, pp. 1-95, 2013.

[15] Y. Ephraim and N. Merhav, "Hidden Markov processes," IEEE Trans. Inf. Theory, vol. 48, no. 6, pp. 1518-1569, Jun. 2002.

[16] Y. Ephraim and W. J. J. Roberts, "An EM algorithm for Markov modulated Markov processes," IEEE Trans. Signal Process., vol. 57, no. 2, pp. 463-470, 2009.

[17] M. Feder, E. Weinstein, and A. V. Oppenheim, "A new class of sequential and adaptive algorithms with applications to noise cancellation," in Proc. Int. Conf. Acoust., Speech, Signal Process., Apr. 1988, vol. 1, pp. 557-560.

[18] J. D. Ferguson, J. D. Ferguson, Ed., "Variable duration models for speech," in Proc. Symp. Appl. Hidden Markov Models Text Speech, Princeton, NJ, USA, 1980, pp. 143-179.

[19] G. R. Grimmett and D. R. Stirzaker, Probability and Random Processes. Oxford, U.K.: Oxford Science, 2001.

[20] Y. Guédon, "Estimating hidden semi-Markov chains from discrete sequences," J. Comput. Graphic. Statist., vol. 12, no. 3, pp. 604-639, 2003.

[21] U. Holst and G. Lindgren, "Recursive estimation in mixture models with Markov regime," IEEE Trans. Inf. Theory, vol. 37, pp. 1683-1690, Nov. 1991.

[22] J. G. Kemeny and J. L. Snell, Finite Markov Chains, 3rd ed. New York, NY, USA: Springer-Verlag, 1983.

[23] P. Lanchantin, J. Lapuyade-Lahorgue, and W. Pieczynski, "Unsupervised segmentation of triplet Markov chains hidden with long-memory noise," Signal Process., vol. 88, no. 5, pp. 1134-1151, 2008.

[24] P. Lanchantin, J. Lapuyade-Lahorgue, and W. Pieczynski, "Unsupervised segmentation of randomly switching data hidden with non-Gaussian correlated noise," Signal Process., vol. 91, no. 2, pp. $163-175,2011$.
[25] J. Lapuyade-Lahorgue and W. Pieczynski, "Unsupervised segmentation of hidden semi-Markov non stationary chains," Signal Process., vol. 92, no. 1, pp. 29-42, 2012.

[26] G. Latouche and V. Ramaswami, Introduction to Matrix Analytic Methods in Stochastic Modeling. Philadelphia, PA, USA: ASA/SIAM, 1999.

[27] F. LeGland and L. Mevel, "Recursive estimation in hidden Markov models," in Proc. 36th IEEE Conf. Decision Control, 1997, vol. 4, pp. 3468-3473.

[28] B. G. Leroux, "Maximum-likelihood estimation for hidden Markov models," Stoch. Process. Appl., vol. 40, pp. 127-143, 1992.

[29] B. L. Mark and Y. Ephraim, "Explicit causal recursive estimators for continuous-time bivariate Markov chains," IEEE Trans. Signal Process., vol. 62, pp. 2709-2718, May 2014.

[30] G. Mongillo and S. Deneve, "Online learning with hidden Markov models," Neural Comput., vol. 20, pp. 1706-1716, 2008.

[31] M. F. Neuts, Matrix-Geometric Solutions in Stochastic Models. Baltimore, MD, USA: The Johns Hopkins Univ. Press, 1981.

[32] T. Nguyen, B. L. Mark, and Y. Ephraim, "Spectrum sensing using a hidden bivariate Markov model," IEEE Trans. Wireless Commun., vol. 12, pp. 4582-4591, 2013.

[33] W. Pieczynski, "Pairwise Markov chains," IEEE Trans. Pattern Anal. Mach. Intell., vol. 25, no. 5, pp. 634-639, 2003.

[34] W. Pieczynski, C. Hulard, and T. Veit, "Triplet Markov chains in hidden signal restoration," presented at the SPIE's Int. Symp. Remote Sens., Crete, Greece, 2002.

[35] M. Rudemo, "State estimation for partially observed Markov chains," J. Math. Anal. Appl., vol. 44, no. 3, pp. 581-611, 1973.

[36] T. Rydén, "Consistency and asymptotically normal parameter estimates for hidden Markov models," Ann. Statist., vol. 22, no. 4, pp. 1884-1895, 1994.

[37] T. Rydén, "On recursive estimation for hidden Markov models," Stoch. Process. Appl., vol. 66, pp. 79-96, 1997.

[38] C. Stiller and G. Radons, "Online estimation of hidden Markov models,” IEEE Signal Process. Lett., vol. 6, no. 8, pp. 213-215, 1999.

[39] Y. Sun, B. L. Mark, and Y. Ephraim, "Online parameter estimation for temporal spectrum sensing," IEEE Trans. Wireless Commun., 2014, submitted for publication.

[40] S. Z. Yu, "Hidden semi-Markov models," Artif. Intell., vol. 174, no. 2 , pp. 215-243, 2010.

[41] S.-Z. Yu and H. Kobayashi, "Practical implementation of an efficient forward-backward algorithm for an explicit-duration hidden Markov model," IEEE Trans. Signal Process., vol. 54, no. 5, pp. 1947-1951, May 2006.

[42] O. Zeitouni and A. Dembo, "Exact filters for the estimation of the number of transitions of finite-state continuous-time Markov processes," IEEE Trans. Inf. Theory, vol. 34, no. 4, pp. 890-893, 1988.

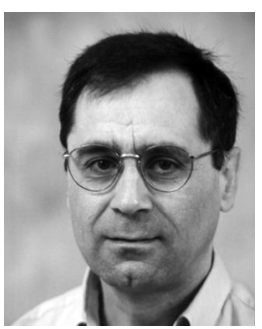

Yariv Ephraim (S'82-M'84-SM'90-F'94) received the D.Sc. degree in electrical engineering from the Technion-Israel Institute of Technology, Haifa, Israel, in 1984. From 1984-1985 he was a Rothschild Postdoctoral Fellow at the Information Systems Laboratory, Stanford University, Stanford, CA. From 1985 to 1993 he was a Member of Technical Staff at the Information Principles Research Laboratory, AT\&T Bell Laboratories, Murray Hill, NJ. In 1991 he joined George Mason University, Fairfax, VA, where he currently is Professor of Electrical and Computer Engineering. His research interests are in statistical signal processing.

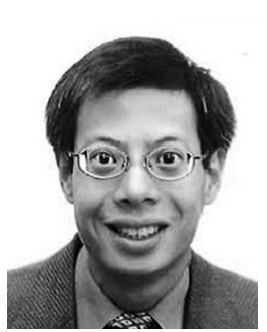

Brian L. Mark (S'91-M'95-SM'08) received the Ph.D. in Electrical Engineering from Princeton University, in 1995 and the B.A.Sc. degree in Computer Engineering with an option in Mathematics from the University of Waterloo in 1991. He was a Research Staff Member at the C\&C Research Laboratories, NEC USA, from 1995 to 1999. In 1999, he was on part-time leave from NEC as a visiting researcher at Ecole Nationale Supérieure des Télécommunications in Paris, France. In 2000, he joined George Mason University, where he is currently Professor of Electrical and Computer Engineering. He served as an Associate Editor for IEEE TRANSACTIONS ON VEHICULAR TECHNOLOGY from 2006-2009. His main research interests lie in modeling and performance analysis of communication networks. 